SISTEMA
ELETRONNICO
DE REVISTAS
SER I UFPR

\title{
Espécies arbóreas de uso múltiplo e sua importância na conservação da biodiversidade nas áreas verdes urbanas de Palmas, Tocantins
}

\section{Arboreal species of multiple use and its importance in the conservation of biodiversity in the urban green areas of Palmas, Tocantins}

\author{
Renato Torres PINHEIRO ${ }^{1 *}$, Dianes Gomes MARCELINO², Dieyson Rodrigues MOURA² \\ ${ }^{1}$ Universidade Federal do Tocantins (UFT), Palmas, TO, Brasil. \\ ${ }^{2}$ Ecótono Engenharia, Palmas, TO, Brasil. \\ *E-mail de contato: renaxas@hotmail.com
}

Artigo recebido em 7 de maio de 2018, versão final aceita em 23 de novembro de 2018.

RESUMO: Palmas/TO foi projetada para ser uma capital ecológica, conciliando a ocupação humana com a preservação de recursos, dentre eles os florestais. Cada uma de suas quadras possui layout específico, pré-determinando as áreas passíveis de serem edificadas e as não edificantes. Entretanto, os recursos florestais desses espaços não edificantes sofrem pressões antrópicas colocando em risco sua conservação e manutenção. Neste sentido, realizamos o inventário qualiquantitativo da arborização das áreas verdes das quadras residenciais da cidade de Palmas com o intuito de identificar a ocorrência de espécies de usos múltiplos, conhecer o seu potencial, valorizar o seu uso pela população e por meio do conhecimento coletivo garantir a sua conservação. $\mathrm{O}$ levantamento foi realizado nas áreas não edificantes de 33 quadras residenciais de Palmas. No total foram identificadas 182 espécies florestais, sendo $54,4 \%$ nativas do Cerrado brasileiro. Mais de 79,0\% das espécies apresentam algum tipo de uso medicinal, $66,5 \%$ são atrativas para a fauna de vertebrados e invertebrados e 40,7\% delas tem emprego na alimentação humana. Observa-se que as áreas não edificantes apresentam grande importância na preservação desses recursos, garantindo o fornecimento de alimento para humanos e animais, além de princípios ativos diversos com potencial para uso medicinal.

Palavras-chave: arborização urbana; recurso florestal; cidades sustentáveis; biodiversidade.

ABSTRACT: The city of Palmas-TO was designed to be an ecological capital, conciliating the human occupation with the preservation of natural resources. Each of its blocks has a specific layout, pre-determining the areas that can be built and those that are not uplifting. However, the forest resources of these non-uplifting spaces suffer 
anthropogenic pressures, putting their conservation and maintenance at risk. In this sense, we carried out the quantitative inventory of the afforestation of the Palmas city's residential blocks green areas of in order to identify the occurrence of species of multiple uses, to know its potential, to value its use by the population and to promote their conservation. The survey was carried out in the non-uplifting areas of 33 residential blocks of Palmas. In total, 182 forest species were identified, of which 54.4\% were native to the Brazilian savanna. More than $79 \%$ of the species presented some type of medicinal use, $66.5 \%$ are attractive to the fauna of vertebrates and invertebrates and $40.7 \%$ of them are edible. It is observed that the non-uplifting areas of the residential blocks of Palmas are of significant importance in the preservation of these resources, guaranteeing the supply of food for humans and animals, as well as diverse active principles with potential for medicinal use.

Keywords: urban afforestation; forest resources; sustainable cities; biodiversity.

\section{Introdução}

O Cerrado brasileiro é uma região prioritária para conservação em nível global e um dos cinco hotspots de biodiversidade da América do Sul (Joppa et al., 2011). Seu reconhecimento e importância como provedor de serviços ecossistêmicos é cada vez maior devido à sua capacidade em armazenar carbono, produzir água e pela elevada diversidade biológica, comparável aos ecossistemas florestais (Overbeck et al., 2015). Por outro lado, o Cerrado brasileiro é um ecossistema profundamente ameaçado em função do incremento das atividades humanas, principalmente aquelas associadas à conversão da terra pelo agronegócio (Fernandes et al., 2016), o que têm contribuído diretamente para o êxodo rural e o crescimento das cidades nos últimos 50 anos (Miccolis et al., 2014).

$\mathrm{O}$ crescimento populacional e a expansão das áreas urbanas também têm sido considerados como fontes de ameaça à conservação da biodiversidade em diferentes regiões do Cerrado como no entorno de Brasília/DF (Diniz \& Brito, 2013) ou Palmas/ TO (Reis et al., 2012). Atualmente, mais de $85 \%$ da população brasileira vive nas cidades e à medida que as áreas urbanas crescem, aumenta a demanda por recursos externos (água, energia, alimentos). Com isso amplificam-se os impactos ambientais diretos, com a conversão e redução de áreas naturais, sobreuso dos recursos hídricos, introdução de espécies exóticas, incremento da poluição do ar e da água, perda de biodiversidade, entre outros (Fernandes et al., 2016).

A proteção dos recursos naturais nos ambientes urbanizados exerce um papel importante na conservação da biodiversidade e manutenção dos serviços ecossistêmicos, melhorando a qualidade do ambiente e de vida da população. Neste cenário, a arborização urbana apresenta-se como um elemento diferenciado na paisagem em função dos inúmeros bens e serviços que repercutem positivamente do ponto de vista social, ambiental, econômico e cultural para a população (McPherson et al., 2016). Diferentes estudos têm demonstrado o importante papel das florestas urbanas na redução dos níveis de poluição e gases de efeito estufa (Nowak et al., 2006; Paoletti, 2009; Zhao et al., 2010; Baró et al., 2014) ou da infraestrutura verde na promoção da saúde em áreas urbanas (Tzoulas et al., 2007).

O Cerrado apresenta a mais rica flora dentre os ambientes savânicos, contendo aproximadamente 4,8\% das espécies de plantas mundiais (Ratter et 
al., 1997), cujo potencial de uso alimentício, artesanal, ornamental, melífero, oleaginoso, medicinal, lactífero, aromático, sendo ainda pouco conhecido e subutilizado (Fernandes et al., 2016). A cidade de Palmas, capital do Tocantins foi planejada dentro de uma perspectiva humanístico-ecológica, tendo a preservação dos recursos naturais como um dos pilares na sua concepção, neste plano, as áreas verdes presentes nos fundos de vale seriam protegidas e cada quadra teria um percentual de área verde não edificante, com o intuito de proporcionar um ambiente mais agradável e com melhor qualidade de vida para a população (Teixeira, 2009). Porém, com o início da sua construção em 1989, observou-se a supressão parcial ou total de extensas áreas de vegetação nativa e a implantação de espécies arbóreas exóticas ao Cerrado, inclusive nas áreas verdes das quadras urbanizadas (Adorno \& Fighera, 2005). Atualmente Palmas/TO é a capital brasileira com maior taxa de crescimento geométrico (IBGE, 2017) e já apresenta um déficit de arborização apesar de sua pouca idade (Prefeitura Municipal de Palmas, 2015). Tendo em vista a presença de remanescentes de Cerrado nativo e as pressões sofridas pela vegetação no meio urbano, realizamos o inventário qualiquantitativo da arborização das áreas verdes das quadras residenciais da cidade com o intuito de identificar a ocorrência de espécies de usos múltiplos e conhecer o seu potencial, de modo a garantir a sua conservação.

\section{Material e métodos}

A cidade de Palmas/TO está situada na região central do Estado do Tocantins e localizada no paralelo $10^{\circ} 11^{\prime} 04^{\prime}$ 'Sul e do meridiano 48 20'01' Oeste.
Fundada em maio de 1989, possui atualmente uma população estimada de 286.787 habitantes (IBGE, 2017), sendo a última cidade brasileira planejada no século XX. O clima na região é quente, sendo caracterizado por duas estações bem definidas: uma chuvosa, entre os meses de outubro a abril; e outra seca, entre os meses de maio a setembro. $O$ índice pluviométrico varia de 1.500 a $1.900 \mathrm{~mm} / \mathrm{ano}$. A temperatura média anual é de $26^{\circ} \mathrm{C}$, sendo setembro o mês mais quente, com média máxima de $36^{\circ} \mathrm{C}$ e julho o mais frio, com média mínima de $15,5^{\circ} \mathrm{C}$. A vegetação natural de Palmas/TO é típica do Cerrado havendo fisionomias florestais (cerradão e mata de galeria), campestres (campo sujo), e savânicas (cerrado sensu stricto) que formam a paisagem local (Barros et al., 2012).

A exemplo de outras cidades planejadas, Palmas/TO está estruturada em áreas residenciais, comerciais e industriais. As quadras residenciais possuem dimensões médias de 700x700m e foram projetadas para abrigar de 8 a $12 \mathrm{mil} /$ habitantes, sendo dotadas de parcelamento interno próprio e planejadas como unidades básicas para organização da vida (Teixeira, 2009). Cada quadra em geral possui escolas, unidades básicas de saúde, áreas comerciais, áreas para esporte e lazer e áreas verdes não edificantes.

No presente estudo, foram consideradas áreas verdes, aquelas presentes no memorial descritivo de cada quadra analisada: as áreas verdes não edificantes, áreas verdes de esporte e lazer e áreas públicas municipais (GeoPalmas, 2017). Os dados foram coletados entre fevereiro e dezembro de 2015, em 33 das 63 quadras residenciais urbanizadas e asfaltadas nas quatros regiões da cidade. As quadras foram escolhidas aleatoriamente e de maneira proporcional ao número de quadras de 
cada região: Área Residencial Sudoeste - ARSO: 205S, 207S, 303S, 403S, 405S, 505S, 603S, 1005S e 1105S; Área Residencial Sudeste - ARSE: 204S, 210S, 306S, 308S, 404S, 504S, 606S e 704S; Área Residencial Noroeste - ARNO: 105N, 303N, 305N, 403N, 405N e Área Residencial Nordeste - ARNE: $106 \mathrm{~N}, 110 \mathrm{~N}, 112 \mathrm{~N}, 208 \mathrm{~N}, 304 \mathrm{~N}, 404 \mathrm{~N}, 406 \mathrm{~N}$, $504 \mathrm{~N}, 506 \mathrm{~N}, 604 \mathrm{~N}$ e $608 \mathrm{~N}$ (Figura 1).

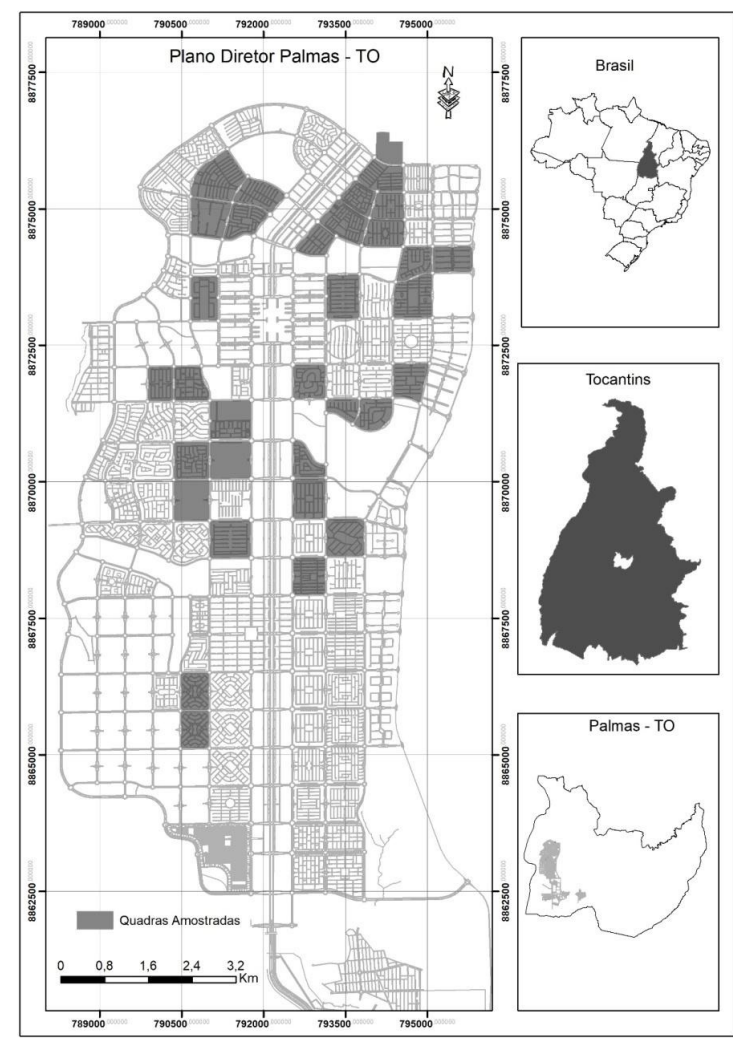

FIGURA 1 - Esquema do Plano Diretor de Palmas, TO, evidenciando as quadras residências amostradas.

FONTE: Instituto de Planejamento Urbano de Palmas (2007). Adaptado por Dieyson Moura.
A coleta de dados foi realizada em campo, mediante o preenchimento de planilha eletrônica digital incorporada em um coletor de dados Mesa Juniper System ${ }^{\circledR}$. Foram coletados dados de todas as árvores presentes nas áreas verdes das quadras, com respeito à espécie (nome científico e popular) e sua origem, sendo classificadas em: $(\mathrm{N})$ nativas do Cerrado, espécies com ocorrência comprovada no bioma Cerrado, (EC) espécies nativas brasileiras, mas com ocorrência fora do bioma Cerrado e (EB) espécies originárias de outros países. Para cada categoria foram obtidos dados referentes à riqueza de espécies e seus usos: para alimentação (A) humana na forma de frutos, sementes, condimentos; medicinais $(\mathrm{M})$, como fitoterápicos ou remédios caseiros; e para a fauna (F) de vertebrados e invertebrados por meio da dispersão de propágulos e polinização, assim foram descritos com base na literatura.

As espécies vegetais foram identificadas em campo. No caso de dúvidas foram coletadas amostras de ramos, flores e/ou frutos, preparas exsicatas e encaminhadas para identificação por especialistas no Herbário da Universidade Federal do Tocantins, Campus de Porto Nacional. Informações taxonômicas das espécies brasileiras foram obtidas na base de dados do Jardim Botânico do Rio de Janeiro (Flora do Brasil, 2017) e Lorenzi (2002; 2013; 2016) e das espécies estrangeiras em The Plant List (2017).

\section{Resultados}

Nas áreas verdes das quadras residenciais de Palmas/TO, foram amostrados 11.498 indivíduos arbóreos de 44 famílias e 182 espécies. Quanto à origem, $54,4 \%$ das espécies e $64,7 \%$ dos indivíduos arbóreos são nativos do Cerrado; $11,5 \%$ das espécies 
e $8,7 \%$ das árvores são brasileiras exóticas ao Cerrado e $34,1 \%$ das espécies e $26,7 \%$ das árvores são espécies estrangeiras, provenientes de outros países.

As espécies arbóreas representaram 88,5\% da amostra e as palmeiras 11,5\%. As espécies mais abundantes foram o Pequi (Caryocar brasiliense Cambess), com 11,7\% do total de árvores, o Caju (Anacardium occidentale L.), com 10,6\% e a Manga (Mangifera indica L.), com $8,8 \%$ do total amostrado. Dentre as palmeiras destacou-se o Coco-da-bahia (Cocos nucifera L.), com 1,6\% do total de indivíduos amostrados (Tabela 1). Das 99 espécies nativas do Cerrado, 44,5\% são utilizadas na alimentação humana, 77,8\% consideradas úteis para a fauna e $82,8 \%$ possuem propriedades medicinais (Guarim Neto et al., 2003; Lorenzi, 2002; 2013; 2016; Oliveira et al., 2010; Kuhlmann, 2012; Silva et al., 2015; Kuhlmann \& Ribeiro, 2016).

TABELA 1 - Famílias, espécies, nome popular, origem e categorias de usos das árvores encontradas nas áreas verdes das quadras urbanizadas de Palmas/TO, durante levantamentos realizados em 2015. Quanto à origem as árvores foram classificadas em nativas do Cerrado (N), exóticas ao Cerrado (EC) e exóticas estrangeiras (EB). As categorias de usos foram: alimentação (A), fauna (F) e medicinal (M).

\begin{tabular}{|c|c|c|c|c|c|c|c|}
\hline Família & Nome Cientifico & Nome Popular & Origem & Total & \multicolumn{3}{|c|}{ Categorias de Usos } \\
\hline \multirow{6}{*}{ Anacardiaceae } & $\begin{array}{c}\text { Anacardium microcarpum } \\
\text { Ducke }\end{array}$ & Cajuí & $\mathrm{N}$ & 68 & $\mathrm{x}$ & $\mathrm{x}$ & $\mathrm{x}$ \\
\hline & Anacardium occidentale $L$. & Caju & $\mathrm{N}$ & 1217 & $\mathrm{x}$ & $\mathrm{x}$ & $\mathrm{x}$ \\
\hline & $\begin{array}{c}\text { Myracrodruon urundeuva } \\
\text { Allemão }\end{array}$ & Aroeira & $\mathrm{N}$ & 24 & $\mathrm{x}$ & $\mathrm{x}$ & $\mathrm{x}$ \\
\hline & $\begin{array}{l}\text { Schinus terebinthifolius } \\
\text { Raddi }\end{array}$ & Aroeira-vermelha & $\mathrm{N}$ & 11 & $\mathrm{x}$ & $\mathrm{x}$ & $\mathrm{x}$ \\
\hline & Spondias purpurea L. & Siriguela & $\mathrm{EB}$ & 20 & $\mathrm{x}$ & $\mathrm{x}$ & $\mathrm{x}$ \\
\hline & $\begin{array}{c}\text { Spondias venulosa (Engl.) } \\
\text { Engl. }\end{array}$ & Cajá & $\mathrm{EC}$ & 5 & $\mathrm{x}$ & $\mathrm{x}$ & $\mathrm{x}$ \\
\hline \multirow{6}{*}{ Annonaceae } & Annona classiflora Mart. & Araticum & $\mathrm{N}$ & 117 & $\mathrm{x}$ & $\mathrm{x}$ & $\mathrm{x}$ \\
\hline & Annona coriacea Mart. & Marolo & $\mathrm{N}$ & 19 & $\mathrm{x}$ & $\mathrm{x}$ & $\mathrm{x}$ \\
\hline & Annona glabra L. & Araticum-dobrejo & $\mathrm{N}$ & 1 & $\mathrm{x}$ & $\mathrm{x}$ & $\mathrm{x}$ \\
\hline & Annona muricata L. & Graviola & EB & 2 & $\mathrm{x}$ & $\mathrm{x}$ & $\mathrm{x}$ \\
\hline & Annona squamosa L. & Pinha & $\mathrm{EC}$ & 119 & $\mathrm{x}$ & $\mathrm{x}$ & $\mathrm{x}$ \\
\hline & $\begin{array}{c}\text { Xylopia aromatica (Lam.) } \\
\text { Mart. }\end{array}$ & $\begin{array}{l}\text { Pimenta-de- ma- } \\
\text { caco }\end{array}$ & $\mathrm{N}$ & 13 & $\mathrm{x}$ & $\mathrm{x}$ & $\mathrm{x}$ \\
\hline
\end{tabular}




\begin{tabular}{|c|c|c|c|c|c|c|c|}
\hline \multirow{7}{*}{ Apocynaceae } & $\begin{array}{c}\text { Aspidosperma macrocarpon } \\
\text { Mart. }\end{array}$ & $\begin{array}{l}\text { Guatambu-do- cer- } \\
\text { rado }\end{array}$ & $\mathrm{N}$ & 26 & & $\mathrm{x}$ & $\mathrm{x}$ \\
\hline & Hancornia speciosa Gomes & Mangaba & $\mathrm{N}$ & 67 & $\mathrm{x}$ & $\mathrm{x}$ & $\mathrm{x}$ \\
\hline & $\begin{array}{l}\text { Himatanthus obovatus } \\
\text { (Müll. Arg.) Woodson }\end{array}$ & Pau-de-leite & $\mathrm{N}$ & 8 & $\mathrm{x}$ & $\mathrm{x}$ & $\mathrm{x}$ \\
\hline & Plumeria alba L. & $\begin{array}{c}\text { Jasmim-manga- } \\
\text {-branco }\end{array}$ & EB & 1 & & $\mathrm{x}$ & $\mathrm{x}$ \\
\hline & Plumeria pudicaJacq. & Buque-de- noiva & EB & 5 & & $\mathrm{x}$ & $\mathrm{x}$ \\
\hline & Plumeria rubra L. & Jasmim-manga & EB & 6 & & $\mathrm{x}$ & $\mathrm{x}$ \\
\hline & $\begin{array}{c}\text { Thevetia peruviana (Pers.) } \\
\text { K.Schum. }\end{array}$ & $\begin{array}{l}\text { Chapéu-de-Napo- } \\
\text { leão }\end{array}$ & $\mathrm{N}$ & 22 & & $\mathrm{x}$ & $\mathrm{x}$ \\
\hline Araliaceae & $\begin{array}{l}\text { Didymopanax morototonii } \\
\text { (Aubl.) Decne\& Planch. }\end{array}$ & Mandioqueiro & $\mathrm{N}$ & 8 & & $\mathrm{x}$ & $\mathrm{x}$ \\
\hline \multirow{11}{*}{ Arecaceae } & $\begin{array}{l}\text { Acrocomia aculeata (Jacq.) } \\
\text { Lodd. ex Mart. } 1845\end{array}$ & Macaúba & $\mathrm{N}$ & 29 & $\mathrm{x}$ & $\mathrm{x}$ & $\mathrm{x}$ \\
\hline & $\begin{array}{c}\text { Attalea phalerata Mart. ex } \\
\text { Spreng. }\end{array}$ & Bacuri & $\mathrm{N}$ & 4 & $\mathrm{x}$ & $\mathrm{x}$ & $\mathrm{x}$ \\
\hline & $\begin{array}{l}\text { Attalea speciosa Mart. ex } \\
\text { Spreng. }\end{array}$ & Babaçu & $\mathrm{N}$ & 5 & $\mathrm{x}$ & $\mathrm{x}$ & $\mathrm{x}$ \\
\hline & $\begin{array}{l}\text { Bismarckia nobilis Hilde- } \\
\text { brant \& Wendland }\end{array}$ & Palmeira-azul & EB & 4 & & & $\mathrm{x}$ \\
\hline & Caryota urens L. & $\begin{array}{l}\text { Palmeira-rabo- de- } \\
\text {-peixe }\end{array}$ & EB & 2 & & $\mathrm{x}$ & $\mathrm{x}$ \\
\hline & Cocos nucifera L. & Coco-da-bahia & $\mathrm{EC}$ & 181 & $\mathrm{x}$ & $\mathrm{x}$ & $\mathrm{x}$ \\
\hline & $\begin{array}{l}\text { Dypsis decaryi (Jum.) Been- } \\
\text { tje\& J. Dransf. }\end{array}$ & Palmeira-triangular & EB & 5 & & & \\
\hline & $\begin{array}{l}\text { Dypsis lutescens (H.Wendl.) } \\
\text { Beentje\&J.Dransf. }\end{array}$ & Areca-bambu & EB & 5 & & $\mathrm{x}$ & $\mathrm{x}$ \\
\hline & $\begin{array}{l}\text { Dypsis madagascariensis } \\
\text { (Becc.) Beentje \& J. Dransf. }\end{array}$ & Areca-locuba & $\mathrm{EB}$ & 42 & & & \\
\hline & Euterpe oleracea Mart. & Açai & $\mathrm{N}$ & 12 & $\mathrm{x}$ & $\mathrm{x}$ & $\mathrm{x}$ \\
\hline & Mauritia flexuosa L.f. & Buriti & $\mathrm{N}$ & 1 & $\mathrm{x}$ & $\mathrm{x}$ & $\mathrm{x}$ \\
\hline
\end{tabular}




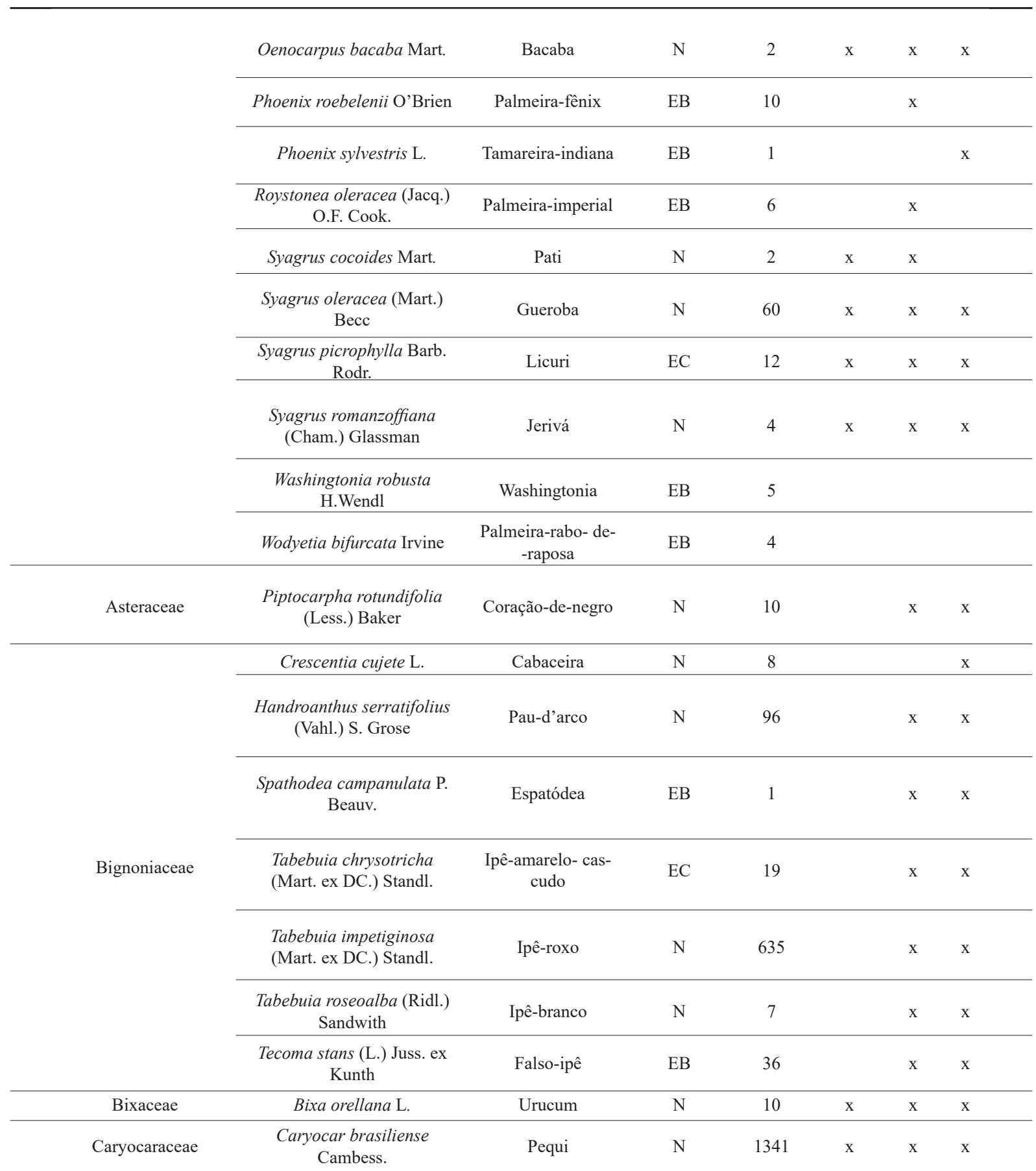




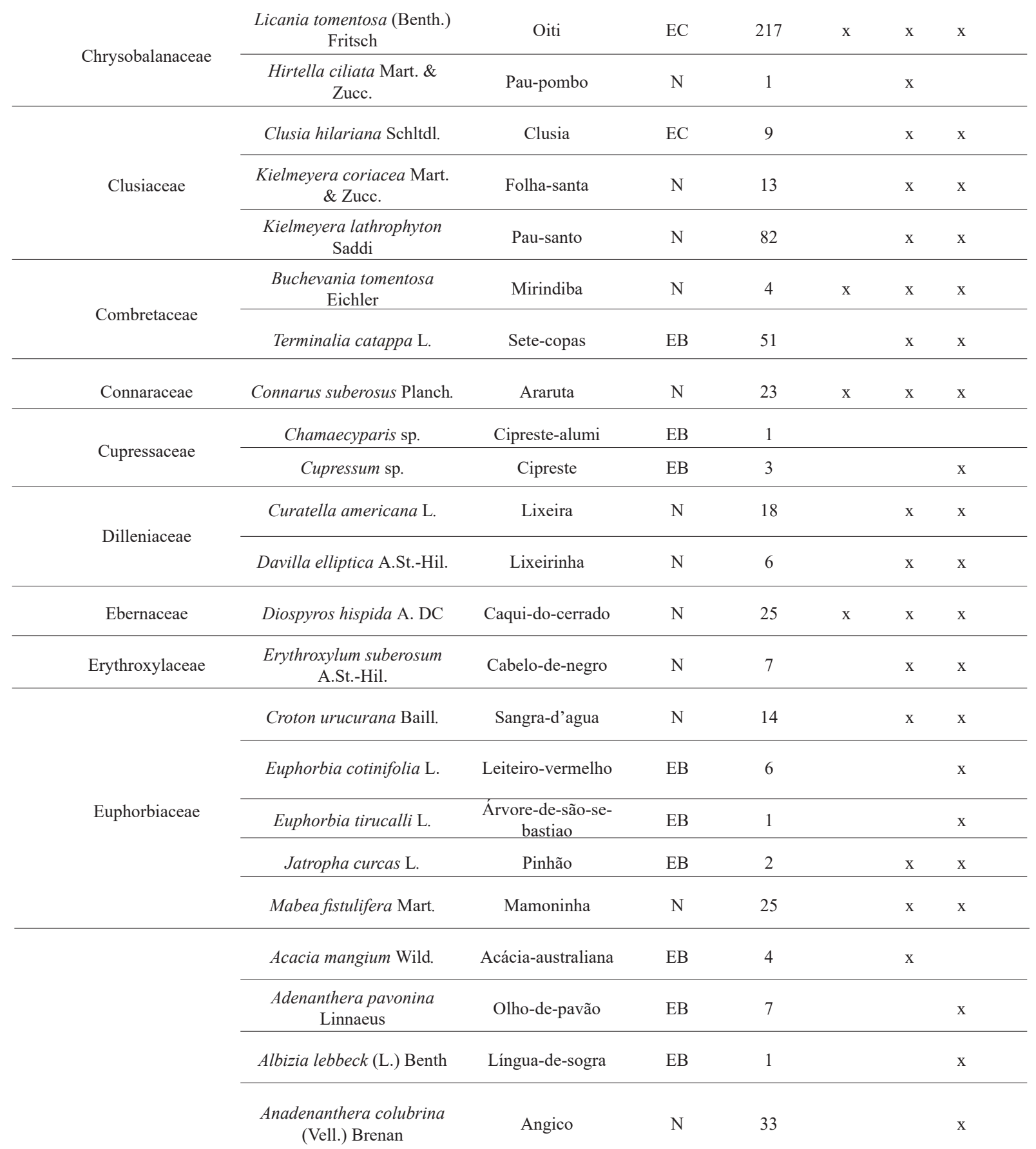




\begin{tabular}{|c|c|c|c|c|c|c|}
\hline Andira cuyabensis Benth. & Fruta-de-morcego & $\mathrm{N}$ & 64 & & $\mathrm{x}$ & $\mathrm{x}$ \\
\hline Andira legalis (Vell.) & Angelim-doce & $\mathrm{N}$ & 45 & & $\mathrm{x}$ & $\mathrm{x}$ \\
\hline Andira vermifuga Mart. & Angelim & $\mathrm{N}$ & 42 & & $\mathrm{x}$ & $\mathrm{x}$ \\
\hline Bauhinia purpurea $\mathrm{L}$. & Pata-de-vaca-roxa & EB & 1 & & & $\mathrm{x}$ \\
\hline Bauhinia variegata $\mathrm{L}$. & Pata-de-vaca & EB & 32 & & & $\mathrm{x}$ \\
\hline $\begin{array}{l}\text { Bowdichia virgilioides } \\
\text { Kunth }\end{array}$ & Sucupira-preta & $\mathrm{N}$ & 117 & & $\mathrm{x}$ & $\mathrm{x}$ \\
\hline Caesalpinia echinata Lam. & Pau-brasil & $\mathrm{EC}$ & 11 & & $\mathrm{x}$ & $\mathrm{x}$ \\
\hline $\begin{array}{c}\text { Caesalpinia peltophoroides } \\
\text { Benth. }\end{array}$ & Sibipiruna & $\mathrm{EC}$ & 5 & & $\mathrm{x}$ & $\mathrm{x}$ \\
\hline $\begin{array}{c}\text { Caesalpinia pulcherrima } \\
\text { (L.) Sw. }\end{array}$ & Flamboyanzinho & EB & 6 & & $\mathrm{x}$ & $\mathrm{x}$ \\
\hline Cassia fistula $\mathrm{L}$. & Canafistula & EB & 19 & & $\mathrm{x}$ & $\mathrm{x}$ \\
\hline $\begin{array}{c}\text { Cenostigma tocantinum } \\
\text { Ducke }\end{array}$ & Pau-preto & $\mathrm{N}$ & 13 & & & \\
\hline Copaifera langsdorffii Desf. & Copaíba & $\mathrm{N}$ & 11 & & $\mathrm{x}$ & $\mathrm{x}$ \\
\hline $\begin{array}{c}\text { Dalbergia miscolobium } \\
\text { Benth. }\end{array}$ & $\begin{array}{l}\text { Jacarandá-do-cer- } \\
\text { rado }\end{array}$ & $\mathrm{N}$ & 24 & & & \\
\hline $\begin{array}{l}\text { Delonix regia (Bojer ex } \\
\text { Hook.) Raf. }\end{array}$ & Flamboyant & EB & 23 & & $\mathrm{x}$ & $\mathrm{x}$ \\
\hline Dimorphandra mollis Benth. & Fava-d'anta & $\mathrm{N}$ & 97 & & $\mathrm{x}$ & $\mathrm{x}$ \\
\hline Dipteryx alata Vogel & Baru & $\mathrm{N}$ & 13 & $\mathrm{x}$ & $\mathrm{x}$ & $\mathrm{x}$ \\
\hline $\begin{array}{l}\text { Enterolobium contortisili- } \\
\text { quum (Vell.) Morong. }\end{array}$ & Tamboril & $\mathrm{N}$ & 22 & & & $\mathrm{x}$ \\
\hline $\begin{array}{l}\text { Enterolobium gummiferum } \\
\text { (Mart.) J.F.Macbr. }\end{array}$ & Orelha-de-negro & $\mathrm{N}$ & 7 & & $\mathrm{x}$ & $\mathrm{x}$ \\
\hline Hymenaeacourbaril L. & Jatobá-da-mata & $\mathrm{N}$ & 18 & $\mathrm{x}$ & $\mathrm{x}$ & $\mathrm{x}$ \\
\hline $\begin{array}{l}\text { Hymenaea stigonocarpa } \\
\text { Mart. ex Hayne }\end{array}$ & Jatobá-do-cerrado & $\mathrm{N}$ & 51 & $\mathrm{x}$ & $\mathrm{x}$ & $\mathrm{x}$ \\
\hline Inga capitata Desv. & Ingá-ferro & $\mathrm{N}$ & 19 & $\mathrm{x}$ & $\mathrm{x}$ & $\mathrm{x}$ \\
\hline Inga edulis Mart. & Ingá-de-metro & $\mathrm{N}$ & 39 & $\mathrm{x}$ & $\mathrm{x}$ & $\mathrm{x}$ \\
\hline
\end{tabular}




\begin{tabular}{|c|c|c|c|c|c|c|c|}
\hline & Inga laurina (Sw.) Willd. & Ingá-branco & $\mathrm{N}$ & 17 & $\mathrm{x}$ & $\mathrm{x}$ & $\mathrm{x}$ \\
\hline & $\begin{array}{l}\text { Leucaena leucocephala } \\
\text { (Lam.) de Wit }\end{array}$ & Leucena & $\mathrm{EB}$ & 46 & & & $\mathrm{x}$ \\
\hline & $\begin{array}{l}\text { Libdibia ferrea (Mart. ex } \\
\text { Tul.) L.P.Queiroz }\end{array}$ & Pau-ferro & $\mathrm{EC}$ & 46 & & & $\mathrm{x}$ \\
\hline & $\begin{array}{c}\text { Machaerium acutifolium } \\
\text { Vogel }\end{array}$ & $\begin{array}{l}\text { Jacarandá-bico-de- } \\
\text {-papagaio }\end{array}$ & $\mathrm{N}$ & 4 & & & $\mathrm{x}$ \\
\hline & $\begin{array}{c}\text { Mimosa caesalpiniifolia } \\
\text { Benth. }\end{array}$ & Sansão-do-campo & $\mathrm{N}$ & 11 & & & $\mathrm{x}$ \\
\hline & Parkia platycephala Benth. & Fava-de-bolota & $\mathrm{N}$ & 200 & & $\mathrm{x}$ & $\mathrm{x}$ \\
\hline & $\begin{array}{l}\text { Plathymenia reticulata } \\
\text { Benth. }\end{array}$ & Vinhático & $\mathrm{N}$ & 132 & & $\mathrm{x}$ & $\mathrm{x}$ \\
\hline & $\begin{array}{c}\text { Pterodon polygalaeflorus } \\
\text { Benth. }\end{array}$ & Sucupira-lisa & $\mathrm{N}$ & 31 & & & $\mathrm{x}$ \\
\hline & $\begin{array}{l}\text { Schizolobium parahyba } \\
\text { (Vell.) Blake }\end{array}$ & Guapuruvu & $\mathrm{EC}$ & 4 & & $\mathrm{x}$ & $\mathrm{x}$ \\
\hline & $\begin{array}{l}\text { Senna siamea (Lam.) } \\
\text { H.S.Irwin \& Barneby }\end{array}$ & Sena & EB & 104 & & & $\mathrm{x}$ \\
\hline & Tachigali aurea Tul. & Carvoeiro & $\mathrm{N}$ & 41 & & & \\
\hline & $\begin{array}{l}\text { Tachigali rubiginosa (Mart. } \\
\text { ex Tul.) Oliveira-Filho }\end{array}$ & Cachamorra & $\mathrm{N}$ & 526 & & & \\
\hline & Tamarindus indica $\mathrm{L}$. & Tamarindo & EB & 51 & $\mathrm{x}$ & & $\mathrm{x}$ \\
\hline & $\begin{array}{l}\text { Vatairea macrocarpa (Ben- } \\
\text { th.) Ducke }\end{array}$ & Amargoso & $\mathrm{N}$ & 191 & & $\mathrm{x}$ & $\mathrm{x}$ \\
\hline Lamiaceae & Aegiphila lhotskiana cham. & Tamanqueiro & $\mathrm{N}$ & 1 & & & $\mathrm{x}$ \\
\hline \multirow{2}{*}{ Lauraceae } & $\begin{array}{l}\text { Cinnamomum burmanni } \\
\text { (Ness \& T. Ness) Blume }\end{array}$ & Falsa-canela & EB & 1 & & & \\
\hline & Persea americana Mill. & Abacate & EB & 35 & $\mathrm{x}$ & $\mathrm{x}$ & $\mathrm{x}$ \\
\hline Lecythidaceae & $\begin{array}{c}\text { Cariniana rubra Gardner } \\
\text { ex Miers }\end{array}$ & $\begin{array}{l}\text { Cachimbo-de-ma- } \\
\text { caco }\end{array}$ & $\mathrm{N}$ & 1 & & & $\mathrm{x}$ \\
\hline \multirow{2}{*}{ Lythraceae } & $\begin{array}{c}\text { Physocalymma scaberrimum } \\
\text { Pohl }\end{array}$ & Cega-machado & $\mathrm{N}$ & 54 & & & \\
\hline & Punica granatum $\mathrm{L}$. & Romã & EB & 2 & $\mathrm{x}$ & & $\mathrm{x}$ \\
\hline
\end{tabular}




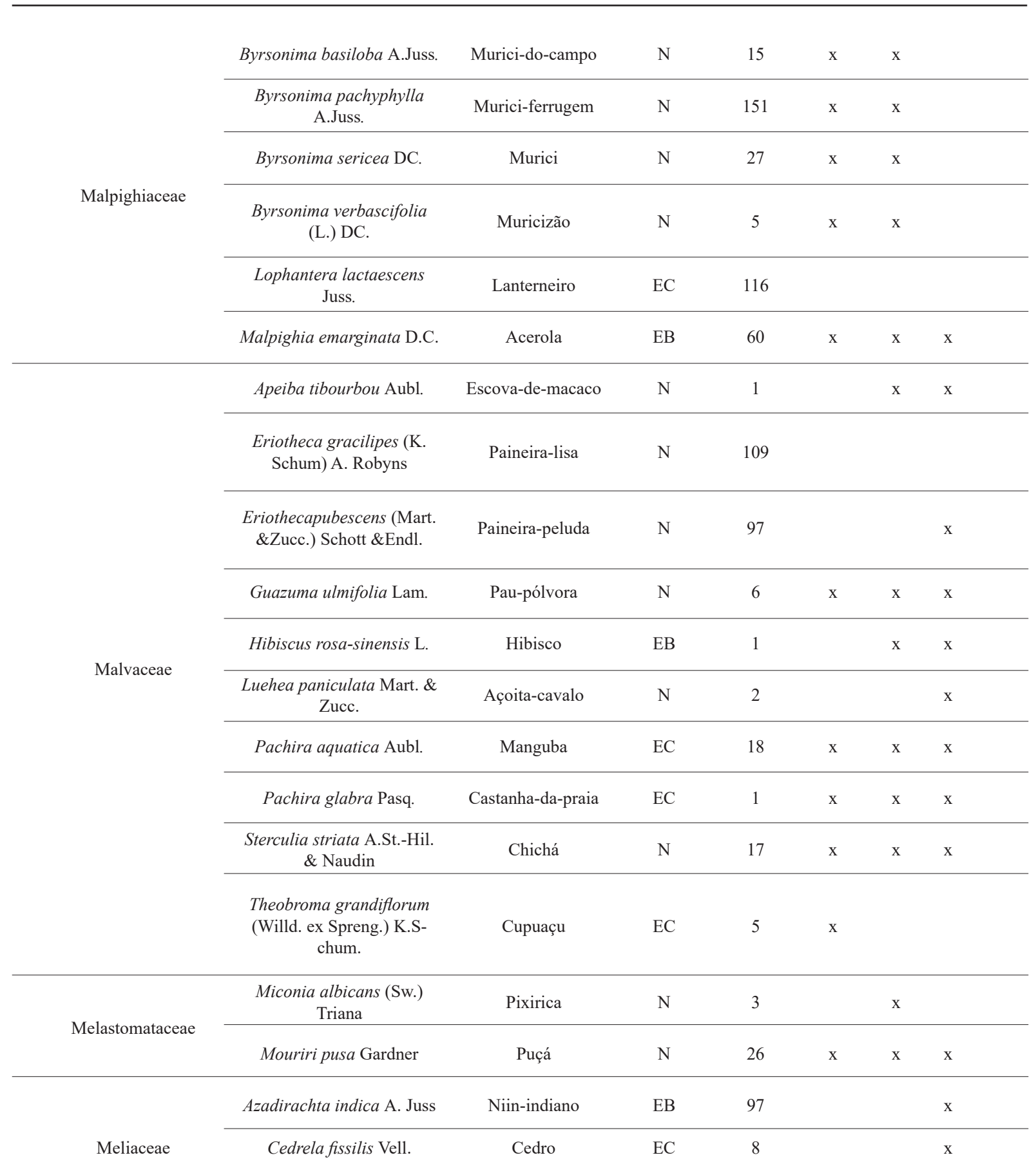




$\begin{array}{lll}\text { Swietenia macrophylla King } \quad \text { Mogno } & \text { EC }\end{array}$

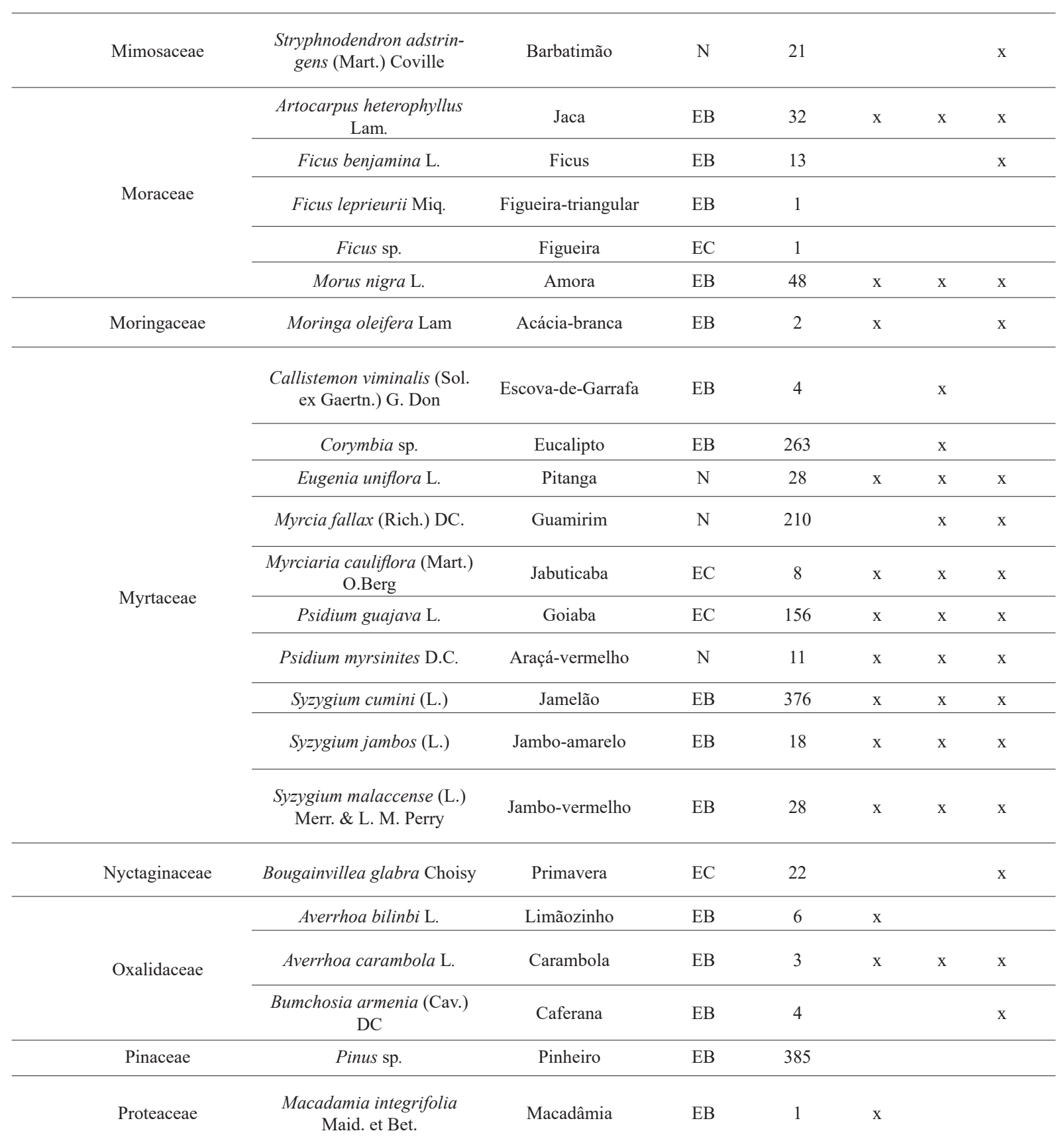




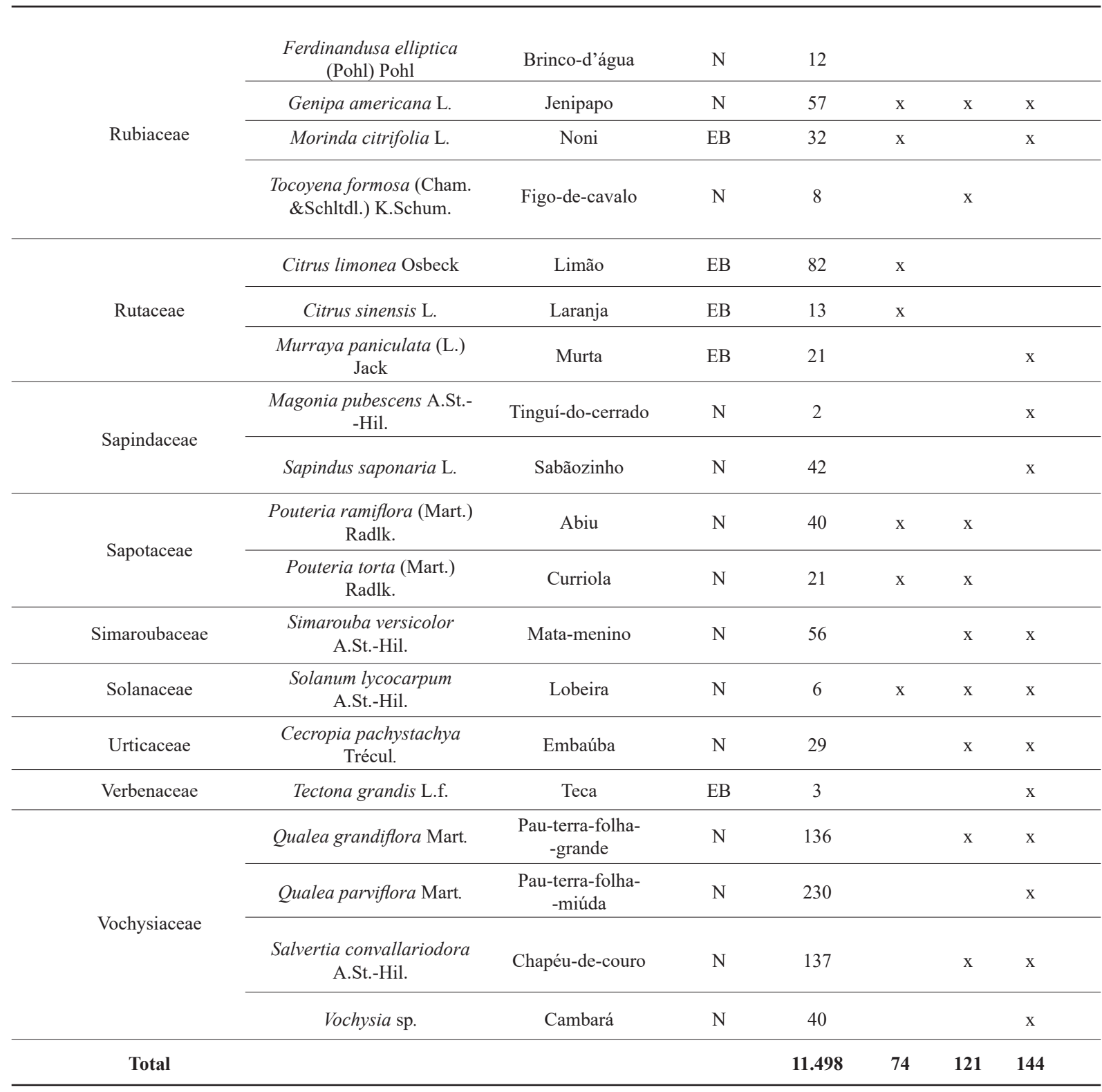


A Figura 2 apresenta a porcentagem de cada uma das categorias de usos das espécies arbóreas encontradas nas áreas verdes das quadras de Palmas/ TO, evidenciando a elevada proporção de espécies úteis descritas na literatura, em cada uma das categorias, especialmente entre aquelas espécies nativas do Cerrado e medicinais.

\section{Discussão}

As espécies nativas do Cerrado presentes nas áreas verdes das quadras residenciais de Palmas/TO se destacaram como as mais importantes do ponto de vista sócio-econômico e ambiental. Preservar as espécies nativas é muito importante, pois, apesar do Cerrado possuir uma flora vascular muito diversa, apenas um número moderado de espécies tem ampla distribuição, havendo uma infinidade de espécies com distribuição restrita (Bridgewater et al., 2004). Além disso, nas formações savânicas do Cerrado, justamente aquelas que predominam nas áreas verdes das quadras urbanizadas de Palmas/ TO, a maior riqueza florística é encontrada no estrato herbáceo-arbustivo (Mendonça et al., 2008), porém esta parte da vegetação é totalmente suprimida para implantação do paisagismo, com consequente perda de espécies arbustivas e arbóreas em estágios iniciais de desenvolvimento. A retirada dos estratos inferiores não é seletiva e ocorre baseada em critérios estéticos e de segurança, pois aumenta a visibilidade nestas áreas públicas.

No caso das espécies exóticas ao Cerrado, estas têm sido implantadas nas áreas verdes pelo poder público municipal ou pela população. De

Alimentação

Fauna

Medicinais

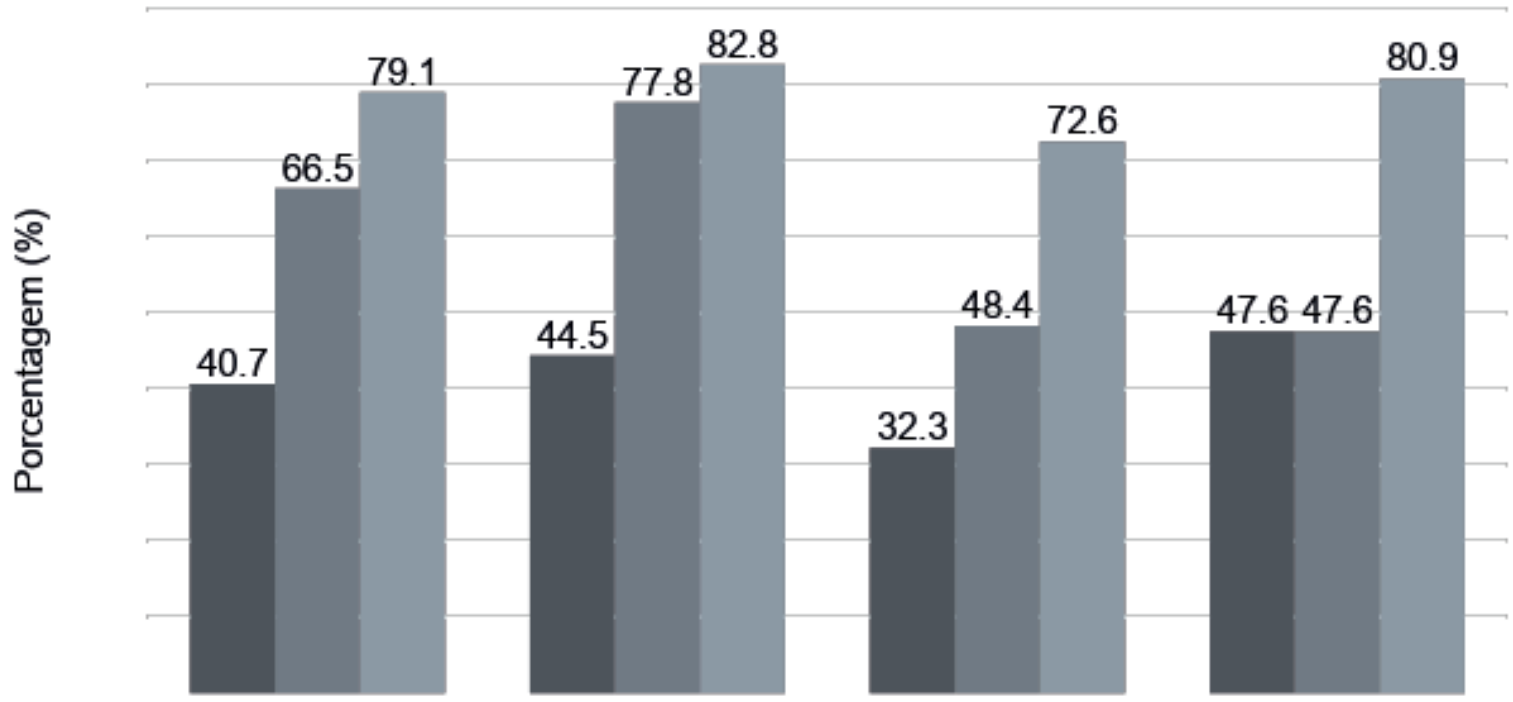

FIGURA 2 - Porcentagem de cada uma das categorias de usos das espécies arbóreas encontradas nas áreas verdes das quadras de Palmas/TO. 
uma maneira geral, as espécies plantadas pelo poder público, são aquelas de fácil propagação ou amplamente utilizadas na arborização urbana como o Oiti (Licania tomentosa (Benth.) Fritsch) ou o Jamelão (Syzygium cumini (L.)). Em contrapartida, a população tende a plantar árvores que de alguma maneira sejam úteis, fornecendo sombra, frutos, flores, o que reforça o caráter social destes ambientes e contribui para a diversificação da arborização urbana (observação pessoal). Por outro lado, algumas espécies exóticas possuem potencial invasor, tornando-se uma grande ameaça para a conservação da biodiversidade local (IUCN, 2000). No Brasil, entorno de 54 espécies vegetais exóticas são consideradas invasivas (Dias et al., 2013). Segundo Biondi \& Leal (2008), nas áreas urbanas, a falta de estudos prévios sobre as espécies e de planejamento na arborização, estão entre os principais fatores responsáveis pela introdução de espécies exóticas invasoras.

Nas áreas verdes a categoria medicinal foi aquela com maior riqueza e abundância de árvores, um padrão também encontrado em diversas outras regiões do Cerrado (Silva et al., 2015). Das 44 famílias, 81,8\% apresentaram importância medicinal, com destaque para a família Fabaceae pela elevada riqueza $(\mathrm{N}=40)$ e onde o percentual de espécies com propriedades medicinais correspondeu a $85 \%$ do total. Silva et al. (2015) também destacaram a importância desta família por sua representatividade no Cerrado mineiro e por seu potencial medicinal. Algumas espécies desta Família, nativas do Cerrado tocantinense, apresentaram elevada frequência nas áreas verdes de Palmas/TO como a Sucupira-preta (Bowdichia virgilioides Kunth), o Vinhático (Plathymenia reticulata Benth.), e o Amargoso (Vatairea macrocarpa (Benth.) Ducke), reconhecidas por sua atividade antimicrobiana e ou antiinflamatória (Guarim Neto \& Morais, 2003).

Pelo menos 270 espécies da flora do Cerrado são consideradas medicinais (Vieira \& Martins, 2000). Nas áreas verdes das quadras de Palmas/ TO, 82,2\% das espécies nativas do Cerrado se enquadram nessa categoria revelando a importância e potencial deste recurso no meio urbano. Ademais, no caso das plantas medicinais, a sua presença na cidade tem importância sócio-cultural, pois permite o seu uso pela população local e proporciona o resgate do conhecimento tradicional que vem sendo perdido pelo êxodo rural e o modo de vida das comunidades urbanas (Oliveira et al., 2010).

Ainda dentro do aspecto sócio-cultural, pouco mais de um terço das espécies arbóreas são potencialmente utlizadas pela população como alimento, muitas delas dotadas de características químicas como antioxidantes e ou elevado valor nutritivo (Rocha et al., 2013). A riqueza de espécies e a elevada abundância de algumas frutíferas nativas do Cerrado como o Pequi (C. brasiliense), com 11,7\% do total de árvores, o Caju (A. occidentale), com $10,6 \%$, o Murici-ferrugem (B. pachyphylla), com $1,31 \%$ e o Araticum (A. classiflora), com 1,02\%, têm oportunizado o seu uso pela população local, aumentando o fluxo de pessoas nas áreas verdes durante a época de frutificação (observação pessoal). Além disso, as espécies frutíferas promovem o resgate de usos tradicionais ou proporcionam novos usos gastronômicos por meio destas fontes alternativas de alimentação (Siqueira, 2012).

Já as frutíferas exóticas ao Cerrado, correspondem a 30 espécies encontradas nas áreas verdes. Nesta categoria existem aquelas plantadas primariamente para consumo das frutas, como a Goiaba (Psidium guajava), a Pitanga (Eugenia uniflora), 
o Côco-da-bahia (Cocos nucifera) ou árvores para sombreamento, mas cujo fruto também pode ser utilizado para alimentação como o Oiti (Licania tomentosa) e o Cacau-selvagem (Pachira aquatica). Dentre as espécies estrangeiras 32,3\% são aproveitadas na alimentação com destaque para a Manga (M. indica), que é a exótica mais abundante, com mais de mil árvores plantadas, o que corresponde a quase $10 \%$ do total de árvores encontradas nas áreas verdes das quadras analisadas.

Cerca de um terço das espécies do Cerrado produzem frutos atrativos para a fauna e aproximadamente metade das espécies de vertebrados do bioma (aves, mamíferos não-voadores e morcegos) se alimenta constantemente ou parcialmente desses frutos (Kuhlmann \& Ribeiro, 2016). Nas áreas verdes das quadras de Palmas/TO aproximadamente $66,5 \%$ das espécies arbóreas são úteis para a fauna de vertebrados e invertebrados. Dentre as espécies nativas, mais de três quartos são atrativas à fauna, o que pode representar um diferencial na manutenção da fauna e das fisionomias naturais do bioma na área urbana estudada.

A fauna desempenha um papel fundamental na polinização e dispersão de propágulos, auxiliando na conservação e perpetuação de diversas espécies vegetais. Ainda que a dispersão possa ser realizada por diversos agentes como o vento e a água, um grande número de espécies vegetais do Cerrado são zoocóricas e dependem da fauna para sua propagação. Aproximadamente 325 espécies vegetais do Cerrado são dispersadas pela fauna e a zoocoria predomina em diversas fitofisionomias do Cerrado, sendo as aves o principal agente dispersor, seguido dos mamíferos (Kuhlmann \& Ribeiro, 2016).

A relação da avifauna com a arborização em Palmas/TO foi estudada por Reis et al. (2012) os quais registraram 135 espécies de aves nas quadras urbanizadas e demonstraram haver um relacionamento positivo entre a riqueza de aves e as espécies arbóreas nativas do Cerrado e negativo quando comparado às especies arbóreas exóticas. Estes autores também identificaram um efeito negativo da urbanização sobre a riqueza de aves frugívoras, sendo esta significativamente menor nas quadras mais urbanizadas e com menor cobertura arbórea nativa do Cerrado. Alterações na estrutura da vegetação influenciam diferencialmente grupos tróficos, e no caso dos frugívoros, grandes responsáveis pela dispersão de propágulos, sua redução pode comprometer a dispersão natural de diversas espécies da flora local. A redução da diversidade arbórea exerce influência direta na eficiência e qualidade dos serviços ambientais, repercutindo na qualidade do ar (Nowak et al., 2006) ou na promoção da saúde (Tzoulas et al., 2007) no meio urbano.

Apesar do importante papel dos mamíferos na dispersão de sementes, este grupo está escassamente representado nas áreas verdes das quadras de Palmas/TO, o que limitaria a dispersão de frutos predominantemente mamaliocóricos como os dos gêneros Annona, Acromia, Attalea, Hymenaea, Enterolobium, Caryocar, Diospyrus, Pouteria, entre outros (Kuhlmann \& Ribeiro, 2016). Estes mesmos autores revelaram ainda que a manutenção da vegetação savânica e campestre depende em parte da fauna das formações florestais do Cerrado. Neste sentido, a conservação dos ambientes florestais ripários na área urbana de Palmas/TO é de grande importância para a manutenção das áreas verdes das quadras onde predominam as formações savânicas.

$\mathrm{O}$ adequado manejo das áreas verdes das quadras urbanizadas, reduzindo a supressão das espécies herbáceo/arbustivas, restringindo a intro- 
dução de espécies exóticas ou a retirada de espécies arbóreas autóctones, traria melhorias significativas para a qualidade ambiental das quadras residenciais de Palmas/TO, incrementando a diversidade biológica e com isso o número de espécies vegetais úteis para a fauna e para a população local. Além disso manteria estes ambientes estruturalmente mais próximos de seu estado natural, aumentando seu valor social, econômico, ambiental e cultural. Outro fator preponderante, diz respeito à conexão das áreas verdes das quadras com as formações florestais ripárias do meio urbano. Neste caso, a gestão deve priorizar a ampliação da cobertura vegetal e a diversidade de espécies que compõe os corredores verdes de acompanhamento viário, elementos fundamentais na estrutura ecológica urbana de Palmas/ TO. Finalmente, compartilhar com a comunidade informações sobre os valores das árvores como ser vivo, sobre os serviços benéficos que a arborização traz para a melhoria da qualidade de vida e da saúde das pessoas, são ações imprescindíveis para conservação do patrimônio arbóreo das áreas verdes.

\section{Conclusão}

As áreas verdes das quadras de Palmas/TO possuem grande importância do ponto de vista socioeconômico e ambiental haja vista a elevada proporção de espécies arbóreas que possuem usos múltiplos, pertencendo a pelo menos duas das categorias aqui consideradas, maximizando os recursos disponíveis no ambiente urbano. Espécies frutíferas como o Pequi (Caryocar brasiliense), o Cajuí (Anacardium microcarpum), os Muricis (Byrsonima sp.), o Araticum (Annona classiflora), o Jatobá-do-cerrado (Hymenaea stignocarpa), a
Mangaba (Hancornia speciosa) ou o Puçá (Mouriri pusa), fazem parte desse conjunto de espécies que complementam a dieta dos moradores, possuem uso medicinal e/ou atraem a fauna, devendo o poder público e a sociedade zelar pela manutenção desses espaços públicos.

\section{Agradecimentos}

Agradecemos à Prefeitura Municipal de Palmas, a Fundação de Apoio Científico e Tecnológico do Tocantins e a Fundação Universidade Federal do Tocantíns pelo apoio prestado.

\section{Referências}

Adorno, L. F. M.; Fighera, D. R. A Trajetória da Política Ambiental de Palmas Enquanto Capital Ecológica. In: Almeida, M. G. de (Org.). Tantos Cerrados Goiânia: Vieira, p. 205-223, 2005.

Baró, F.; Chaparro, L.; Gómez-Baggethun, E.; Langemeyer, J.; Nowak, D. J.; Terradas, J. Contribution of ecosystem servives to air quality and climate change mitigation policies: the case of urban forests in Barcelona, Spain. AMBIO, 43, 466-479, 2014. doi 10.1007/s13280-014-0507-x

Barros, M. A.; Maia, M. A. M., Paiva, A. L. B. Estudo da dinâmica da cobertura e uso da terra do Estado do Tocantins. Fisionomias da faixa centro. Palmas: Seplan/DZE. 292p. 2012.

Biondi, D.; Leal, L. Caracterização das plantas produzidas no Horto Municipal da Barreirinha - Curitiba/PR. REVS$B A U, 3(2), 20-36,2008$.

Bridgewater, S.; Ratter, J. A.; Ribeiro, J. F. Biogeographic patterns $\beta$-diversity and dominance in the Cerrado biome of Brazil. Biodiversity and Conservation, 13, 2295-2318, 2004. 
Dias, J.; da Fonte, M. A.: Baptista, R.; Mantoani, M. C; Holdefer, D. R.; Torezan, J. M. D. Invasive alien plants in Brazil: a nonrestrictive revision of academic works. Natureza e Conservação, 11(1), 31-35, 2013. doi: 10.4322/ natcon.2013.004

Diniz, M. F.; Brito, D. Threats to and viability of the giant anteater, Myrmecophagatridactyla (Pilosa: Myrmecophagidae), in a protected Cerrado remnant encroached by urban expansion in central Brazil. Zoologia, 30(2), 151-156, 2013. doi: 10.1590/S1984-46702013000200005

Fernandes, G. W.; Pedroni, F.; Sanchez, M.; Scariot, A.; Aguiar, L. M. S.; Ferreira, G.; Machado, R.; Ferreira, M. E.; Diniz, S.; Pinheiro, R. T.; Costa, J. A. S.; Dirzo, R.; Muniz, F. Cerrado: em busca de soluções sustentáveis. Rio de Janeiro: Vozes, 2016.

Flora do Brasil. Jardim Botânico do Rio de Janeiro, 2017. Disponível em: <http://floradobrasil.jbrj.gov.br>. Acesso em out. 2017.

GeoPalmas. Sistema de Informações Geográficas de Palmas. Disponível em: $<$ http://geo.palmas.to.gov.br $>$. Acesso em jul. 2017.

Guarim Neto, G.; Morais, R. G. Recursos medicinais de espécies do cerrado de Mato Grosso:um estudo bibliográfico. Acta Botanica Brasilica, 17(4), 561-584, 2003. doi: 10.1590/S0102-33062003000400009

IBGE - Instituto Brasileiro de Geografia e Estatística. Censo demográfico 2010: estimativa populacional para o ano de 2017. 2017. <www.cidades.ibge.gov.br>. Acessoem: out. 2017.

IUCN - International Union for Conservation of Nature. IUCN guidelines for the preservation of biodiversity loss caused by allien invasive species. Fifth Meeting of the Conference of the Parties to the Convention of Biological Diversity. Nairobi, Kenia, 2000.

Joppa, L. N.; Roberts, D. L.; Myers, N.; Pimm, S. L. Biodiversity hotspots house most undiscovered plant species. Proceedings of the National Academy of Sciences of the United States of America, 108(32), 13171-13176, 2011. doi: 10.1073/pnas.1109389108

Kuhlman, M. Frutos e sementes do Cerrado atrativos para a fauna: guia de campo. Brasília, DF: Rede de sementes do Cerrado, 2012.

Kuhlmann, M.; Ribeiro, J. F. Evolution of seed dispersal in the Cerrado biome: ecological and phylogenetic considerations. Acta Botanica Brasilica, 30(2), 271-282, 2016. doi: 10.1590/0102-33062015abb0331

Lorenzi, H. Árvores brasileiras: manual de identificação e cultivo de plantas arbóreasnativas do Brasil. Nova Odessa: Instituto Plantarum de Estudos da Flora. 4. ed., Vol. 1, 2002.

Lorenzi, H. Árvores brasileiras: manual de identificação e cultivo de plantas arbóreas nativas do Brasil. Nova Odessa: Instituto Plantarum de Estudos da Flora. 4 ed., Vol. 2, 2013.

Lorenzi, H. Árvores brasileiras: manual de identificação e cultivo de plantas arbóreas nativas do Brasil. Nova Odessa: Instituto Plantarum de Estudos da Flora. 2 ed., Vol. 3, 2016.

McPherson, E. G.; van Doorn, N.; Goede, J. Structure, function and value of street trees in California, USA. $U r$ ban Forestry \& Urban Greening, 17, 104-115, 2016. doi: 10.1016/j.ufug.2016.03.013

Mendonça, R. C.; Felfili, J. M.; Walter, B. M. T.; Silva Júnior, M. C.; Rezende, A. V.; Filgueiras, T. S.; Nogueira, P. E. Flora vascular do bioma Cerrado. In: Sano, S. M.; Almeida, S. P.; Ribeiro, J. F. (Orgs.). Cerrado: ecologia e flora. Brasília: Embrapa Cerrados. vol. 2, p. 421-1279, 2008.

Miccolis, A.; Andrade, R. M. T.; Pacheco P. Land-use trends and environmental governance policies in Brazil: Paths forward for sustainability. Bogor, Indonesia: CIFOR. 2014.

Nowak, D. J.; Crane, D. E.; Stevens, J. C. Air pollution removal by urban trees and shrubs in the United States. Urban Forestry \& Urban Greening, 4, 115-123, 2006. doi: 10.1016/j.ufug.2006.01.007.

Oliveira, G. L.; Oliveira, A. F. M.; Andrade, L. de H. C. Plantas medicinais utilizadas na comunidade urbana de $\mathrm{Mu}$ ribeca, Nordeste do Brasil. Acta Botânica Brasilica, 24(2), 571-577, 2010. doi: 10.1590/S0102-33062010000200026

Overbeck, G. E.; Vélez-Martin, E.; Scarano, F. R.; Lewinsohn, T. M.; Fonseca, C. R.; Meyer, S. T.; Muller, S. C.; Ceotto, P.; Dadalt, L.; Durigan, G.; Ganade, G.; Gossner, M. M.; Guadagnin, D. L.; Lorenzen, K.; Jacobi, C. M.; Weisser, W. W.; Pillar, V. D. Conservation in Brazil needs to 
include non-forest ecosystems. Diversity and Distributions, 21, 1455-1460, 2015. doi:10.1111/ddi. 12380

Paoletti, E. Ozone and urban forests in Italy. Environmental Pollution, 157, 1506-1512, 2009.

Prefeitura Municipal de Palmas. Diagnóstico da Arborização Urbana de Palmas. Palmas, Tocantins, 348p. 2015.

Ratter, J. A.; Ribeiro, J. F.; Bridgewater, S. The Brazilian Cerrado vegetation and threats to its biodiversity. Annals of Botany, 80(3), 223-230, 1997. doi: 10.1006/anbo.1997.0469

Reis, E.; López-Iborra, G. M.; Pinheiro, R. T. Changes in bird species richness through different levels of urbanization: Implications for biodiversity conservation and garden design in Central Brazil. Landscape Urban Plannig, 107, 31-42, 2012. doi: 10.1016/j.landurbplan.2012.04.009

Rocha, M. S.; Figueiredo, R. W.; Araújo, M. A. da M.; Araújo, R. S. dos R. M. Caracterização físico-química e atividade antioxidante (in vitro) de frutos do cerrado piauiense. Revista Brasileira de Fruticultura, 35(4), 933-941, 2013. doi: 10.1590/S0100-29452013000400003

Silva, A. F.; Rabelo, M. F. R.; Enoque, M. M. Diversidade de angiospermas e espécies medicinais de uma área de Cerrado. Revista Brasileira de Plantas Medicinais, 17(4), 1016-1030, 2015. doi: 10.1590/1983-084x/14_115
Siqueira, J. F. Resgate e inovação no uso da biodiversidade dos cerrados. Pesquisas, Botânica, 63, 219-224, 2012.

Teixeira, L. F. C. A formação de Palmas. Revista UFG, 9(6), 91-99, 2009.

The Plant List. Version 1.1. 2017. <www.theplantlist.org>. Acesso out. 2017.

Tzoulas, K.; Korpela, K.; Venn, S.; Yli-Pelkonen, V.; Kazmierczak, A.; Niemela, J.; James, P. Promoting ecosystem and human health in urban áreas using Green Infrastructure: A literature review. Landscape and Urban Planning, 81,167178, 2007. doi 10.1016/j.landurbplan.2007.02.001

Vieira, R. F.; Martins, M. V. M. Recursos Genéticos de Plantas Medicinais do Cerrado: uma compilação de dados. Revista Brasileira de Plantas Medicinais, 3(1), 13-36, 2000.

Zhao, M.; Kong, Z.; Escobedo, F. J.; Gao, J. Impacts of urban forests on offsetting carbon emissions from industrial energy use in Hangzhou, China. Journal of Environmental Management, 91, 807-813, 2010. 